\title{
Composición por tallas y ciclo reproductivo de Pseudupeneus grandisquamis (Pisces: Mullidae) en el Pacífico central Mexicano
}

\author{
G. Lucano-Ramírez, S. Ruiz-Ramírez \& J.A. Rojo-Vázquez \\ Departamento de Estudios para el Desarrollo Sustentable de Zonas Costeras, Centro Universitario de la Costa Sur, \\ Universidad de Guadalajara, Gómez Farias 82, San Patricio-Melaque, C.P. 48980, Jalisco, México. Tel: (052- \\ 315) 3556330, Fax (052-315) 3556331; lucanorg@costera.melaque.udg.mx; sruizram@costera.melaque.udg.mx y \\ jrojo@costera.melque.udg.mx
}

Recibido 04-II-2005. Corregido 03-VIII-2005. Aceptado 31-VIII-2005.

\begin{abstract}
Size composition and reproductive cycle of Pseudupeneus grandisquamis (Pisces: Mullidae) in the Central Mexican Pacific. From June 1995 to December 1998 we used shrimp trawl nets to capture 492 Pseudupeneus grandisquamis in soft-bottom grounds off the central Mexican Pacific (Jalisco and Colima). The ength-weight ratio indicates allometric growth $\left(p=0.0035 L^{3.46} \mathrm{y} \mathrm{r}^{2}=0.97\right)$. The total sex ratio was 1:1.15 (females: males). Mean total length was $153.8 \mathrm{~mm}$ (range 77-236 mm). A progression in length was found: the highest means were observed at the end of 1996 and during 1997. The mean length of females (162 mm) was longer than in males $(150 \mathrm{~mm})$. Four gonadic maturity stages were observed in both sexes; in females the most frequently stage was stage IV (mature gonads, 48.6\%), while in males it was stage III (gonads in maturation, $45.0 \%$ ). Seven microscopic oocyte development stages were identified. Oocyte development seems to be asynchronous. The mature testicle showed sperm inside the lobular lumen and in the duct. The highest values of the gonadosomatic index, as well as the largest percentages of individuals with mature gonads (at macroscopic and microscopic levels), were observed during winter and summer, suggesting that this species has two reproduction peaks by year. The length at which $50 \%$ of the individuals show gonads in maturation $\left(L_{50}\right)$ was $183 \mathrm{~mm}$ for the females and $181 \mathrm{~mm}$ for the males. Rev. Biol. Trop. 54(1): 195-207. Epub 2006 Mar 31.
\end{abstract}

Key words: Pseudupeneus grandisquamis, Mullidae, fish reproduction, Jalisco, Colima, Mexico.

Pseudupeneus grandisquamis (Gill 1863) forma parte de la ictiofauna demersal de la plataforma continental de Jalisco y Colima, zona que se caracteriza por presentar una alta biodiversidad (Aguilar-Palomino et al. 1996, González-Sansón et al. 1997, Landa-Jaime et al. 1997, Godínez-Domínguez et al. 1998). En el océano Pacífico la especie se distribuye desde California hasta Perú, incluyendo las Islas Galápagos. Se le encuentra sobre fondos arenosos, fangosos o rocosos, cerca de la costa y en aguas profundas, en el Golfo de California se captura con redes de arrastre. Es de hábitos bentónicos y se alimenta de organismos pequeños, los cuales son detectados por barbillones dotados de papilas gustativas que sirven para palpar o escudriñar el fondo. Presenta cuerpo alargado y levemente comprimido, es de color rosa a rojo y presenta una mancha roja oscura poco aparente en la mitad del cuerpo el cual esta cubierto de grandes escamas caedizas, en las aletas predomina el color rosa (Allen y Robertson 1994, Schneider 1995, Froese y Pauly 2005).

Dado al escaso conocimiento que se tiene sobre $P$. grandisquamis, el presente trabajo tiene como objetivo describir algunos aspectos sobre la estructura de tallas y la reproducción que presenta ésta especie en la plataforma continental de Jalisco y Colima, México. 


\section{MATERIALES Y MÉTODOS}

La zona de estudio forma parte de la plataforma continental de Jalisco y Colima, México, desde Punta Farallón en el estado de Jalisco $\left(19^{\circ} 21^{\prime} \mathrm{N}, 105^{\circ} 01^{\prime} \mathrm{W}\right)$ hasta Cuyutlán en el estado de Colima $\left(18^{\circ} 55^{\prime} \mathrm{N}, 104^{\circ} 07^{\prime} \mathrm{W}\right)$. La plataforma continental en esta región es muy angosta. Si se limita a la isóbata de los $200 \mathrm{~m}$, su anchura es de tan sólo 7-10 km. La dinámica de las corrientes costeras se caracteriza por un flujo promedio con dirección noroeste en los meses de verano y en dirección contraria en invierno (De la Lanza-Espino 1991). La marea barotrópica en la laguna de Barra de Navidad origina variaciones en el nivel del mar de hasta $1.2 \mathrm{~m}$. También en la plataforma continental se han detectado mareas con periodo diurno y semidiurno. Por otro lado, la región de estudio en verano y otoño sufre la influencia de ciclones $\mathrm{y}$ tormentas tropicales (Filonov et al. 2000).

Las recolectas de organismos se realizaron a bordo del Barco de Investigación Pesquera (BIP V), propiedad de la Universidad de Guadalajara entre mayo de 1995 y diciembre de 1998 (16 cruceros). El BIP V está equipado para la pesca de arrastre tipo camaronero utilizando dos redes tipo semiportuguesa (abertura de operación $6.9 \mathrm{~m}$, altura calculada de la relinga de $1.15 \mathrm{~m}$ y luz de malla en el copo de $38 \mathrm{~mm}$ ). En todos los cruceros de pesca, los arrastres fueron nocturnos, con una duración de media hora, velocidad de dos nudos con rumbo norte-sur y a diferentes estratos de profundidad $(20,40,60$ y $80 \mathrm{~m})$.

La captura obtenida por cada red (babor y estribor) fue separada, y se preservó en hielo hasta su traslado y posterior procesamiento en las instalaciones del Centro de Ecología Costera de la Universidad de Guadalajara en San Patricio-Melaque, Jalisco. Una mejor descripción de la zona de estudio y los procedimientos de muestreo son explicados por González-Sansón et al. (1997).

A cada organismo se le determinó la longitud total (Lt), con precisión de $1 \mathrm{~mm}$ y el peso total $(\mathrm{Pt})$, con precisión de $0.1 \mathrm{~g}$. Se extrajeron las gónadas, las cuales fueron pesadas $(\mathrm{Pg})$, con precisión de $0.01 \mathrm{~g}$, y se preservaron en formol neutro al $10 \%$ para su posterior procesamiento. El grado de desarrollo de las gónadas se realizó con base en las características macroscópicas que describe Nikolsky (1963). La descripción microscópica del ovario se hizo según lo recomendado por Lucano-Ramírez et al. (2001), mientras que, para la descripción microscópica del testículo se siguió la metodología propuesta por Cole (2002). Se determinó el diámetro de los ovocitos, en sus distintas fases, utilizando una cámara digital Motic acoplada a un microscopio Carl-Zeiss Axiostar.

Se estimaron los parámetros de la relación peso-longitud utilizando la expresión:

$$
\mathrm{Pt}={ }_{\mathrm{a}} \mathrm{Lt}^{\mathrm{b}}(1)
$$

donde: $\mathrm{Pt}=$ peso total , $\mathrm{Lt}=$ longitud total, $\mathrm{a}$ y $\mathrm{b}$ son las constantes de la ecuación de regresión. Se realizó una prueba de $t$ de student (Zar 1996), para determinar si el valor obtenido de $b$, es diferente de tres (valor que representa crecimiento tipo isométrico).

Se determinó la proporción de sexos total, anual y por clase de longitud de talla; se aplicó la prueba de $\mathrm{Ji}^{2}$ para probar posibles diferencias en las proporciones. En todos los casos se utilizó un valor de alfa $=0.05$ (Zar 1996).

Además, mensualmente se determinó la frecuencia de tallas, la talla promedio de hembras y machos, los porcentajes de los estadios de madurez gonádica y el índice gonadosomático (IGS), mediante la expresión:

$$
\mathrm{IGS}=(\mathrm{Pg} /(\mathrm{Pt}-\mathrm{Pg}))^{*} 100(2)
$$

donde: $\mathrm{Pg}=$ peso de la gónada y $\mathrm{Pt}=$ peso total del individuo.

Se calculó la talla crítica, $\mathrm{L}_{50}$, en la cual el $50 \%$ de los organismos ya presentan una definida madurez sexual, como lo establece Echeverría (1987), y se describe como:

$$
\mathrm{P}_{\mathrm{Lt}}=1 / 1+\mathrm{e}^{(a \mathrm{Lt}+b)}
$$

donde: $\mathrm{P}_{\mathrm{Lt}}=$ porcentaje de organismos maduros a una determinada longitud total, 
$\mathrm{Lt}=$ longitud total, $a$ y $b$ constantes de la ecuación de regresión.

\section{RESULTADOS}

Se recolectaron un total de 492 organismos de $P$. grandisquamis, para los cuales la relación peso-longitud estuvo determinada por la ecuación $\mathrm{Pt}=0.0035 \mathrm{Lt}^{3.46}$, con coeficiente de determinación ( $\left.\mathrm{r}^{2}\right)$ de 0.97 (Fig. 1). La prueba $t$ de student indicó que existe diferencia significativa entre el valor de $b$ calculado $(b=3.46)$ y el esperado bajo la premisa de crecimiento isométrico $(b=3)(\mathrm{t}=267.1$, g.l. $=490 \mathrm{p}<0.001)$, lo que determina que $P$. grandisquamis presenta un crecimiento de tipo alométrico.

La distribución de sexos de $P$. grandisquamis de todos los organismos capturados $\mathrm{y}$ para cada uno de los años, no presentó diferencias estadísticamente significativas mediante la prueba de $\mathrm{Ji}^{2}$ (Cuadro 1). Para el total de individuos, la proporción de sexos fue de 1:1.15 hembras por machos. Asimismo, en la proporción sexual por clase de talla no se encontraron diferencias estadísticamente significativas entre los sexos (Cuadro 2); en general, no se observó predominancia en el número de individuos de un sexo sobre el otro en las diferentes clases de talla.

Para la muestra total se observaron tallas que van desde los 77 a $236 \mathrm{~mm}$, con una talla promedio de $153.8 \mathrm{~mm}$; el $95 \%$ de los organismos presentaron tallas entre 94 a $216 \mathrm{~mm}$

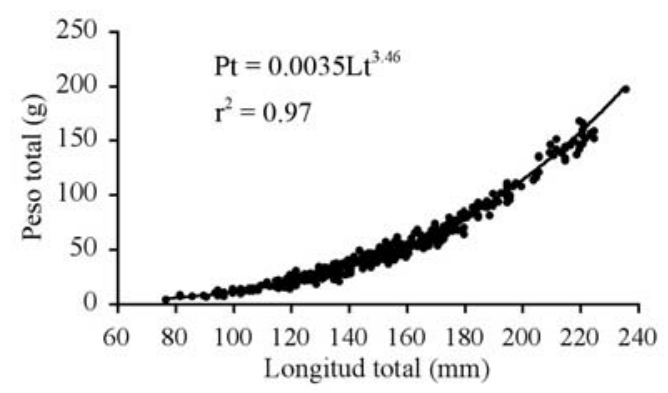

Fig. 1. Relación talla-peso de los organismos capturados con redes de arrastre en las costas de Jalisco y Colima México.

Fig. 1. Length-weight relationship of the organisms captured with trawl nets in the coast of Jalisco and Colima, Mexico.

(Cuadro 3). A su vez, las tallas de hembras y machos presentan solo ligeras diferencias, sin mostrar una notable separación entre estos. En la distribución de tallas de los dos sexos, se puede observar que la forma de las dos distribuciones se aproxima a una distribución normal (Fig. 2), aunque, como es de esperarse, se pueden observar pequeñas diferencias en la frecuencia de tallas entre hembras y machos.

En la distribución mensual de tallas se observa una cierta progresión de las frecuencias de tallas, esto es, las tallas de los organismos tienden a aumentar de acuerdo pasan los meses (Fig. 3). De junio 97 (Jn 97) a mayo 98 (My 98) se presentaron tallas pequeñas, resultado de supuestos eventos reproductivos

CUADRO 1

Valores de tallas de P. grandisquamis que habita las costas de Jalisco y Colima, México

TABLE 1

Values of length of $\mathrm{P}$. grandisquamis that inhabits the coasts of Jalisco and Colima, Mexico

$\begin{array}{lccc} & \text { Hembras } & \text { Machos } & \text { Total } \\ \text { Tamaño de la muestra } & 168 & 190 & 358 \\ \text { Talla promedio (mm) } & 162 & 150 & 155 \\ \text { Intervalo de confianza 95\% } & 100-224 & 88-212 & 94-216 \\ \text { Talla mínima } & 97 & 77 & 77 \\ \text { Talla máxima } & 236 & 222 & 236\end{array}$


CUADRO 2

Proporción sexual anual y total de P. grandisquamis que habita los fondos blandos de las costas de Jalisco y Colima, México

TABLE 2

Annual and total sex ratio of $\mathrm{P}$. grandisquamis that inhabits the soft-bottom of the coast of Jalisco and Colima, Mexico

$\begin{array}{cccc}\begin{array}{c}\text { Año de } \\ \text { muestreo }\end{array} & \text { Hembras } & \text { Hembras: Machos } & \chi^{2} \\ 1995 & 40 & 1.20 & 0.36 \\ 1996 & 96 & 1.04 & 0.04 \\ 1997 & 18 & 1.05 & 0.01 \\ 1998 & 15 & 1.80 & 1.71 \\ \text { Total } & 169 & 1.15 & 2.12\end{array}$

CUADRO 3

Proporción sexual por intervalos de tallas de $20 \mathrm{~mm}$ de P. grandisquamis en la costa de Jalisco y Colima, México

TABLE 3

Sex ratio for intervals of length of $20 \mathrm{~mm}$ of

P. grandisquamis in the coast of Jalisco and Colima, Mexico

$\begin{array}{cccc}\begin{array}{c}\text { Intervalo de } \\ \text { talla }(\mathrm{mm})\end{array} & \text { Hembras } & \begin{array}{c}\text { Hembras: } \\ \text { Machos }\end{array} & \chi^{2} \\ 91-110 & 10 & 2.00 & 1.67 \\ 111-130 & 18 & 1.61 & 1.29 \\ 131-150 & 33 & 1.67 & 2.75 \\ 151-170 & 45 & 0.89 & 0.15 \\ 171-190 & 35 & 0.69 & 1.03 \\ 191-210 & 9 & 1.44 & 0.36 \\ 211-230 & 17 & 0.41 & 2.08\end{array}$

en temporadas anteriores; en junio 98 ( $\mathrm{Jn}$ 98) se presentan de nuevo organismos de tallas medianas, aunque no se observó incremento de estás en el siguiente mes (julio 98); en septiembre 98 (Sp 98) los pocos organismos capturados presentaron tallas mayores a las de julio y agosto del mismo año (Fig. 3).

En cuanto a la distribución mensual de las tallas promedio, para cada uno de los sexos, se observó que a finales de $1996(\mathrm{Nv}-\mathrm{Dc})$ y

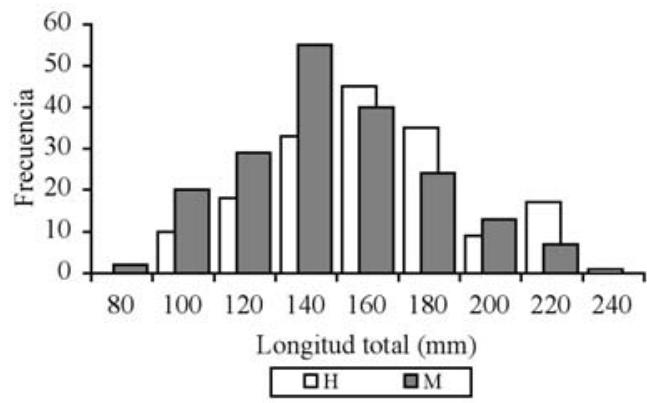

Fig. 2. Frecuencia de tallas de hembras y machos de P. grandisquamis colectados en las costas de Jalisco y Colima, México.

Fig. 2. Length frequencies of females and males of $P$. grandisquamis collected in the coast of Jalisco and Colima, Mexico.

durante los meses de 1997 (En - Sp) se registraron las tallas promedio máximas (Fig. 4). De los 16 meses que se analizaron, en nueve de éstos las hembras presentaron talla promedio mayor a las de los machos, durante cuatro meses los machos presentaron talla promedio mayor que las hembras y en tres meses solo se presento uno de los sexos.

Aunque no se contrastaron las tallas promedio mensuales de hembras y machos, para la mayoría de los meses, la talla promedio de uno $\mathrm{u}$ otro sexo no fue muy diferente; mientras que para toda la muestra, las hembras presentaron una talla promedio ligeramente mayor $(162 \mathrm{~mm})$ a la de los machos (150 mm) (Fig. 4).

En el análisis de las características macroscópicas de las gónadas se observaron cuatro estadios de madurez, según la escala de Nikolsky (1963), tanto para hembras como para machos (inmaduros, en desarrollo, en maduración y maduros) (Fig. 5). Hembras con ovarios inmaduros, se observaron en bajo porcentaje solo en los meses de marzo, abril y julio en 1996. Las hembras con gónadas en desarrollo (estadio II), se presentaron con mayor porcentaje y ocurrieron durante los primeros meses de 1996, en junio y septiembre de 1997 y junio de 1998. A su vez, las hembras con ovarios en maduración (estadio III) se presentaron en los primeros seis meses de 1996, en 

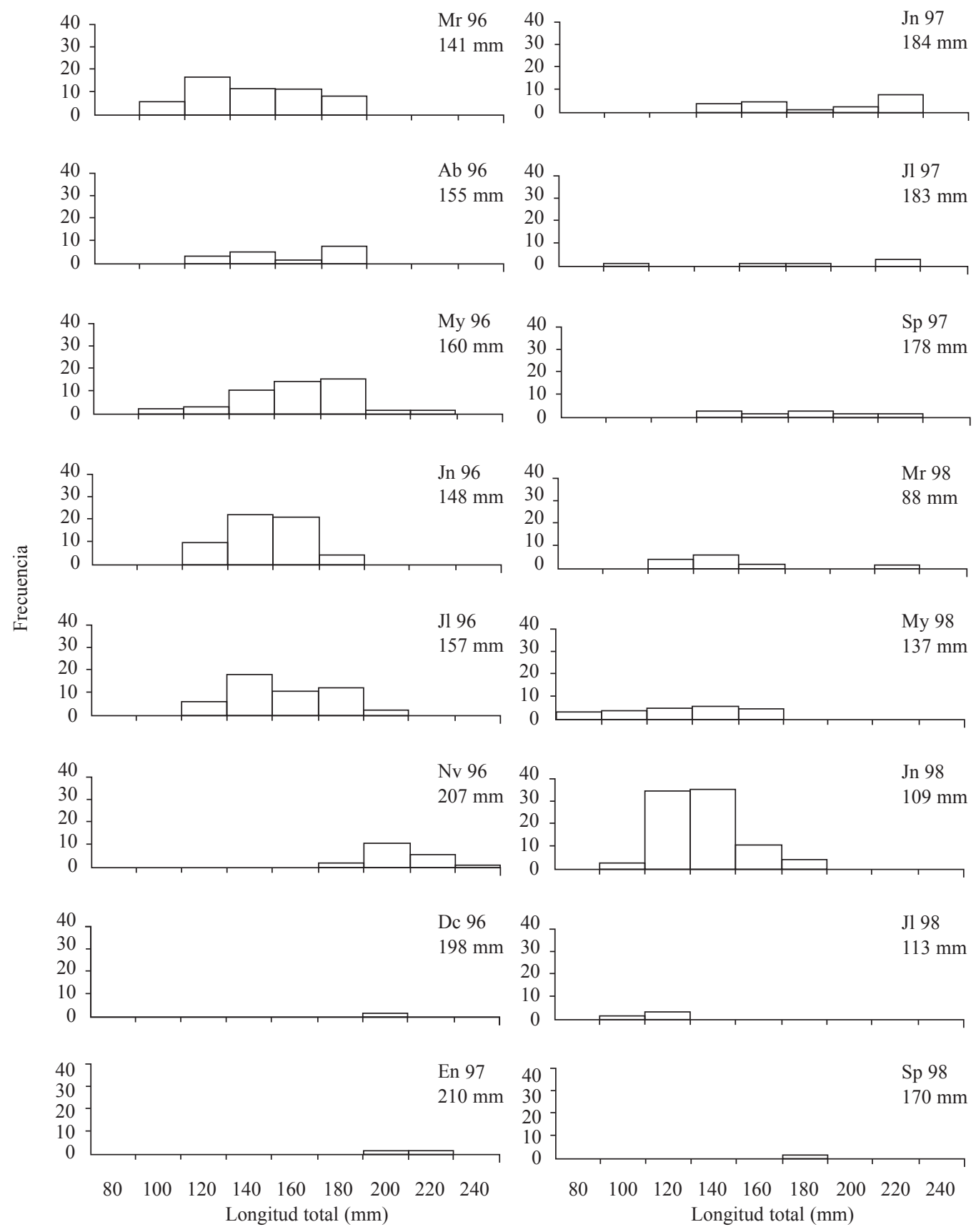

Fig. 3. Frecuencia de tallas de P. grandisquamis en los diferentes meses de muestreo en las costas de Jalisco y Colima, México.

Fig. 3. Length frequencies of $P$. grandisquamis in the different months of sampling in the coast of Jalisco and Colima, Mexico. 


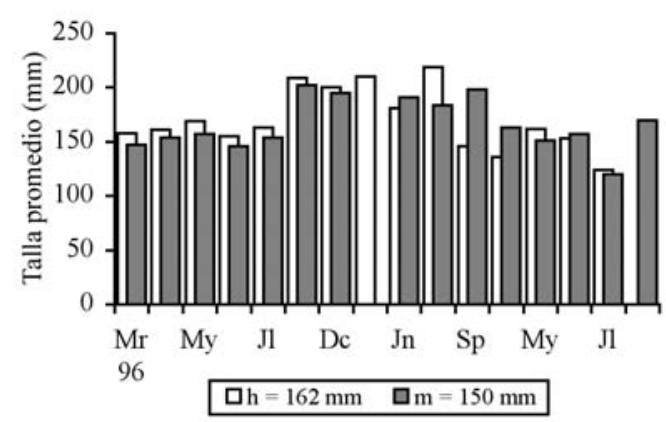

Fig. 4. Talla promedio de hembras (h) y machos (m) de $P$. grandisquamis en los diferentes meses de muestreo en las costas de Jalisco y Colima, México.

Fig. 4. Average length of females (h) and males (m) of $P$. grandisquamis in the different months of sampling in the coast of Jalisco and Colima, Mexico.
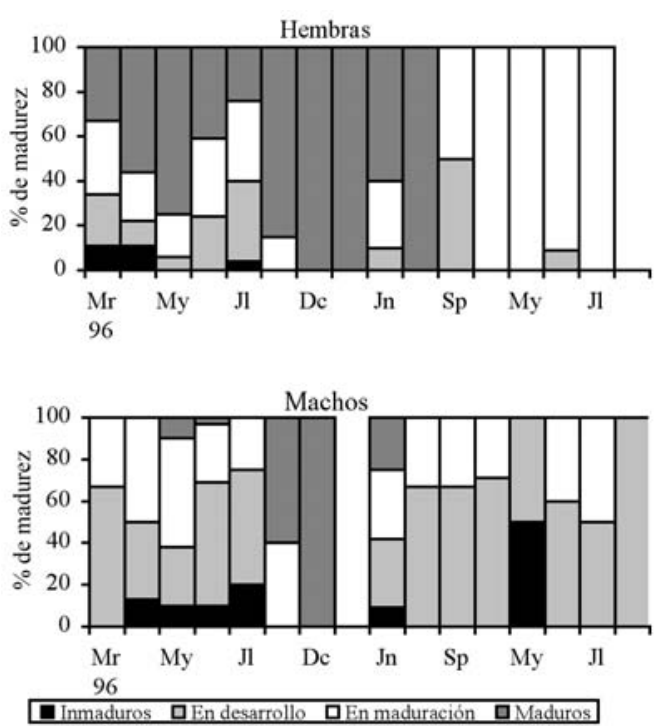

Fig. 5. Porcentaje de los estadios de madurez gonádica de $P$. grandisquamis en los diferentes meses de muestreo en las costas de Jalisco y Colima, México.

Fig. 5. Percentage of the stages of maturity gonadic of P. grandisquamis in the different months of sampling in the coast of Jalisco and Colima, Mexico.

junio, septiembre y octubre de 1997, y en cuatro meses de 1998 (con una ocurrencia entre 90\% y $100 \%)$. Las hembras con ovarios maduros (estadio IV), se presentaron en todos los meses de 1996 y en los tres primeros de 1997 (con un porcentaje de ocurrencia entre el 20-100\%). El periodo reproductivo en las hembras no esta claramente restringido, mas bien parece sugerir que es un proceso con cierta continuidad, aunque con dos picos.

Por otro lado, machos con gónadas inmaduras se observaron en bajo porcentaje alrededor de los meses de mayo y junio de los tres años; machos con gónadas en desarrollo (estadio II), presentaron los mayores porcentajes y ocurrieron en la mayoría de los meses de los tres años; de igual forma machos con testículos en maduración (estadio III) se registraron durante la mayoría de los meses de este estudio; finalmente, los machos con testículos maduros (estadio IV), ocurrieron en bajo porcentaje y su presencia se restringe a los meses de mayo, junio, noviembre y diciembre de 1996, y junio de 1997. De manera general no se observa un periodo bien definido de reproducción, sin embargo, pudiera ser que dicho periodo se concentre durante los meses de mayo-junio y diciembre.

En las hembras, el estadio de madurez gonadal mas abundante es el de gónadas maduras (estadio IV), con un $48.6 \%$, el cual corresponde a individuos con talla promedio de 186 $\mathrm{mm}$; mientras que en los machos el estadio mas frecuente fue el de gónadas en desarrollo (estadio II), con un $45.0 \%$, que corresponde a organismos con talla promedio de $153 \mathrm{~mm}$ (Cuadro 4). Esta diferencia en el grado de madurez de las gónadas entre los dos sexos, podría deberse a la diferencia en las tallas promedio de los mismos sexos, es decir, los machos presentaron gónadas con menor grado de desarrollo que las hembras, debido a que también presentaron menor talla promedio que las hembras.

En el análisis microscópico de ovarios inmaduros (estadio I) se observaron principalmente ovocitos en etapa cromatina nucleolo (Fig. 6a). En los ovarios en desarrollo (estadio II) se distinguen ovocitos en las etapas de cromatina nucleolo, en perinucleolo y con vesículas vitelinas (Fig. 6b). Mientras que en ovarios en maduración (estadio III) se encontraron ovocitos en cromatina nucleolo, en perinucleolo, con vesículas vitelinas, en vitelogénesis 
CUADRO 4

Porcentaje, talla promedio e intervalo de talla al 95\% de hembras y machos de $\mathrm{P}$. grandisquamis en los diferentes estadios de madurez gonádica colectado en las costas de Jalisco y Colima, México

TABLE 4

Percentage, length average and length interval to $95 \%$ of females and males of $\mathrm{P}$. grandisquamis in the different stage of maturity gonadic collected in the coasts of Jalisco and Colima, México

\begin{tabular}{lcccccr} 
& \multicolumn{3}{c}{ Hembras } & \multicolumn{3}{c}{ Machos } \\
Estadios de madurez & $\%$ & Talla (mm) & Intervalo & $\%$ & Talla (mm) & Intervalo \\
Inmaduro (I) & 2.8 & 139 & $76-202$ & 12.1 & 136 & $123-150$ \\
En desarrollo (II) & 8.3 & 152 & $144-161$ & 45.0 & 153 & $147-159$ \\
En maduración (III) & 40.4 & 160 & $153-167$ & 33.3 & 169 & $162-176$ \\
Maduro (IV) & 48.6 & 186 & $179-193$ & 7.6 & 190 & $172-208$
\end{tabular}

primaria y en vitelogénesis secundaria (Fig. 6c). En los ovarios maduros (estadio IV), además de observar las cinco fases antes mencionadas, se observan ovocitos en vitelogénesis terciaria y ovocitos maduros (Fig. 6d).

Esto nos indica que, en los ovarios de P. grandisquamis el desarrollo de los ovocitos es de tipo asincrónico. Se obtuvo el diámetro de las siete fases de desarrollo de los ovocitos encontrados en los ovarios de esta especie (Cuadro 5), y se puede apreciar que, el diámetro promedio de los ovocitos se incrementa a medida que avanza el grado de desarrollo de los mismos.

El testículo presenta un desarrollo de tipo lobular, esto es, dentro de los lóbulos se encuentran grupos de cistos en diferentes fases de desarrollo, y dentro de los cistos se lleva a cabo el desarrollo de las células, pasando desde espermatogonias hasta espermatozoides. En testículos inmaduros (estadio I) y en desarrollo (II) solo se observaron espermatocitos (Fig. 7a). En testículos en maduración (estadio III) se observaron espermatocitos y espermatozoides en poca cantidad en el centro del testículo (Fig. 7b). En los testículos maduros (estadio IV) la cantidad de espermatozoides se incrementa de manera considerable en el centro del testículo (Fig. 7c).

Durante 1996 los máximos valores del índice gonadosomático (IGS) para las hembras se

CUADRO 5

Diámetro de las distintas fases de ovocitos encontrados en el ovario de P. grandisquamis

TABLE 5

Diameter of the different oocyte phases found in the ovary of $\mathrm{P}$. grandisquamis

\begin{tabular}{lccc}
\multicolumn{1}{c}{ Fase de desarrollo de los ovocitos } & N & Media & Intervalo \\
Ovocito Cromatina Nucleolo & 90 & $28.7 \pm 9.8$ & $14.7-56.9$ \\
Ovocito en Perinucleolo & 70 & $52.1 \pm 18.6$ & $27.5-102.2$ \\
Ovocito con Vesículas Vitelinas & 70 & $110.7 \pm 20.9$ & $67.1-154.7$ \\
Ovocito en Vitelogénesis Primaria & 75 & $159.4 \pm 30.6$ & $114.7-149.7$ \\
Ovocito en Vitelogénesis Secundaria & 84 & $228.2 \pm 30.2$ & $170.5-315.7$ \\
Ovocito en Vitelogénesis Terciaria & 30 & $291.5 \pm 24.6$ & $242.3-259.4$ \\
Ovocitos Maduros & 30 & $270.8 \pm 10.7$ & $254.9-297.3$
\end{tabular}



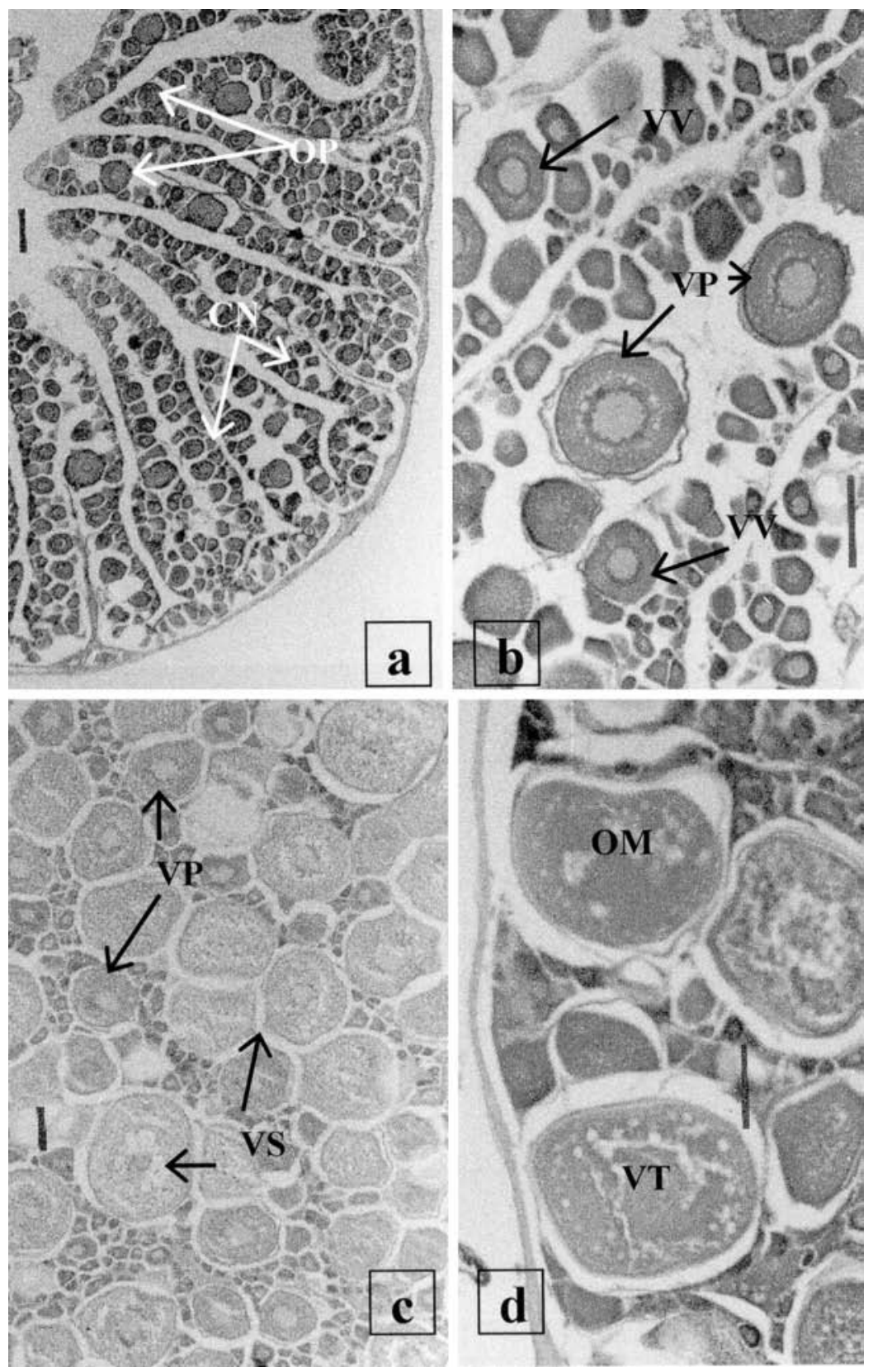

Fig. 6. Cortes de ovario de P. grandisquamis en distintos estadios de madurez mostrando las diferentes fases de desarrollo de los ovocitos. A) Ovario inmaduro; B) Ovario en desarrollo; C) Ovario en maduración y D) Ovario maduro. CN, ovocito en cromatina nucleolo; OP, ovocito en perinucleolo; VV, ovocito con vesículas vitelinas; VP, ovocito en vitelogénesis primaria; VS, ovocito en vitelogénesis secundaria; VT, ovocito en vitelogénesis terciaria; OM, ovocito maduro. Tinción hematoxilina-eosina.

Fig. 6. Sections of ovaries of $P$. grandisquamis in different stages of maturity showing the different phases of development of the oocytes. A) Inmature ovary; B) Ovary in development; C) Ovary in maturation and D) Mature ovary. CN, chromatin nucleolar oocyte; OP, perinucleolar oocyte; VV, yolk vesicles oocyte; VP, primary yolk oocyte; VS, secondary yolk oocyte; VT, tertiary yolk oocyte; OM, mature oocyte. Hematoxylin-eosin stain. 

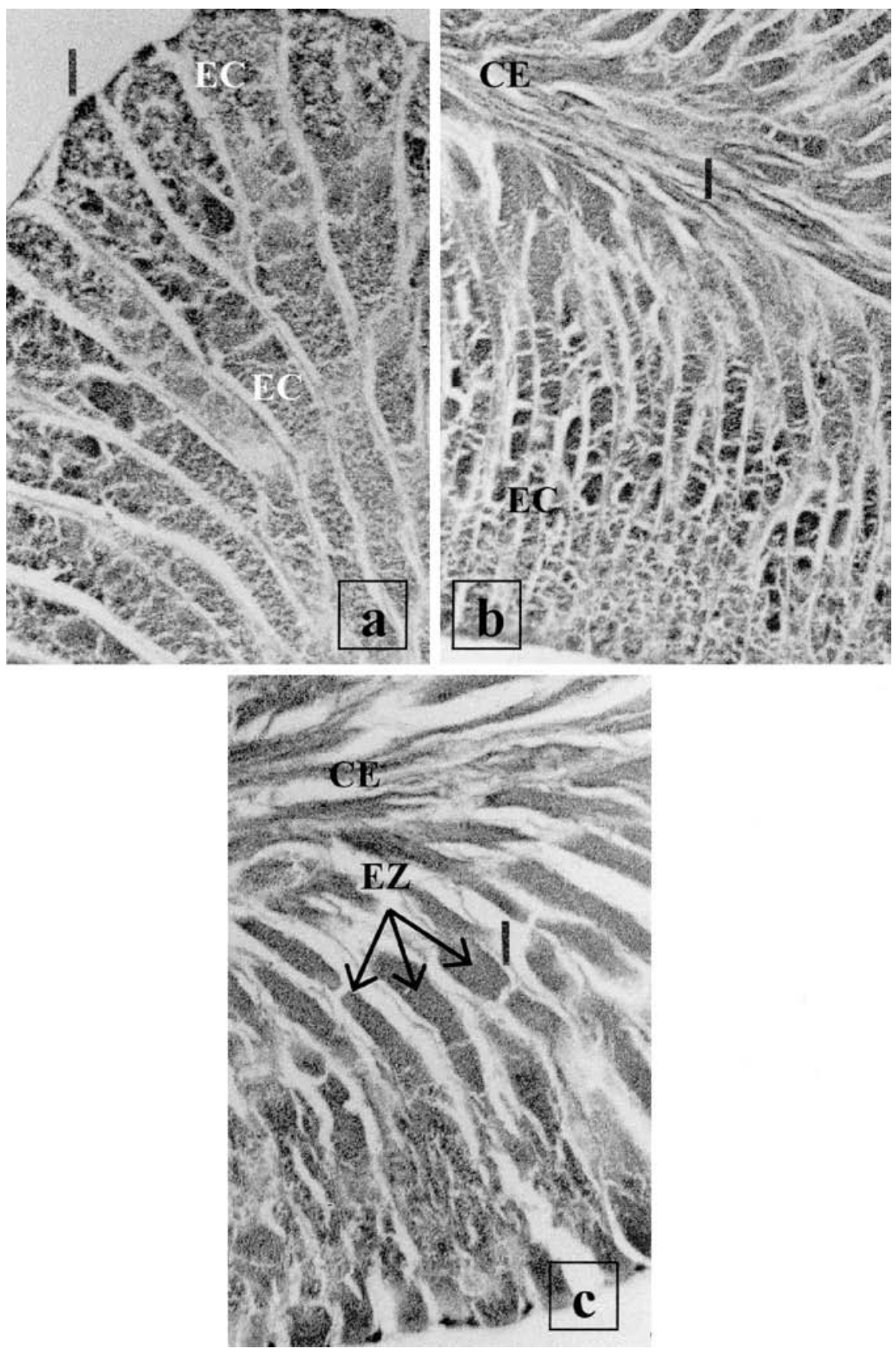

Fig. 7. Cortes de testículo de $P$. grandisquamis en distintos estadios de madurez mostrando el desarrollo de los espermatozoides. A) Testículo en desarrollo; B) Testículo en maduración; C) Testículo maduro. C, cistos; EC, espermatocitos; EZ, espermatozoides; CE, conducto espermático. Tinción hematoxilina-eosina.

Fig. 7. Sections of testes of P. grandisquamis in different stages of maturity showing the development of the sperms. A) Testicle in development; B) Testicle in maturation; C) Mature testicle. C, cyst; EC, spermatocytes; EZ, spermatozoa; CE, espermatic conduct. Hematoxylin-eosin stain. 
presentaron en mayo, noviembre y diciembre. En enero de 1997 el IGS se incrementa (aunque no hay continuidad mensual en los muestreos) y en junio de este año se presenta el máximo valor encontrado para todo el periodo. En 1998 los valores mas altos del IGS se presentaron en los meses de marzo, mayo y julio, aunque estos son de menor magnitud respecto de los años anteriores, representan a los organismos con gónadas maduras (Fig. 8). Es evidente que el IGS en los machos no muestra tantas variaciones como en las hembras, como sucede en muchas especies de peces, sin embargo, existe una ligera correspondencia entre los dos índices, ya que al incrementar el de hembras, el de machos lo hace también, pero a una escala menor (Fig. 8).

La talla crítica, $L_{50}$, para las hembras fue de $183 \mathrm{~mm}$ (Fig. 9), y estuvo descrita por la funcion:

$$
\mathrm{P}_{\mathrm{Lt}}=1 / 1+\mathrm{e}^{(-0.5094 \mathrm{Lt}+9.3079)}
$$

mientras que para los machos la talla crítica fue de 181 mm (Fig. 9), descrita por la funcion:

$$
\mathrm{P}_{\mathrm{Lt}}=1 / 1+\mathrm{e}^{(-0.5777 \mathrm{Lt}+10.436)}(5)
$$

prácticamente no existen diferencias entre ambas tallas.

Con los resultados hasta aquí presentados se puede señalar que $P$. grandisquamis se reproduce principalmente en dos periodos, uno a mediados y otro hacia finales del año. Si bien no se encontraron gónadas en las etapas de reproducción y desove (estadios V y VI, respectivamente), los organismos con gónadas en maduración encontrados podrían proporcionar información confiable, respecto de la temporada reproductiva, ya que en poco tiempo alcanzarían el grado máximo de desarrollo de la gónada y por consecuente el desove de la misma.

\section{DISCUSIÓN}

En los trabajos de Allen y Robertson (1994) y Schneider (1995), la talla máxima descrita

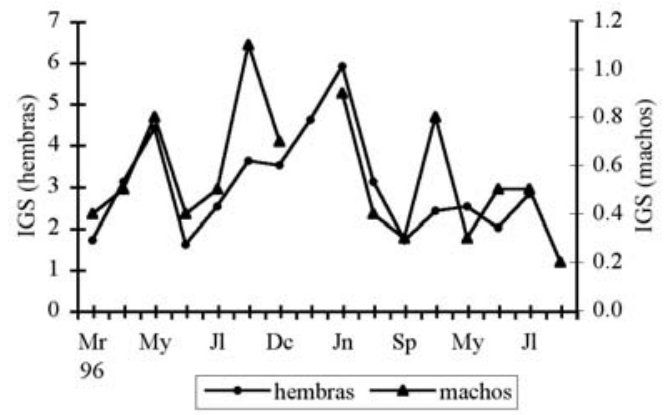

Fig. 8. Índice gonadosomático (IGS) de hembras y machos de $P$. grandisquamis colectados en la costa de Jalisco y Colima, México.

Fig. 8. Gonadosomatic index (IGS) of females and males of $P$. grandisquamis collected in the coast of Jalisco and Colima, Mexico.
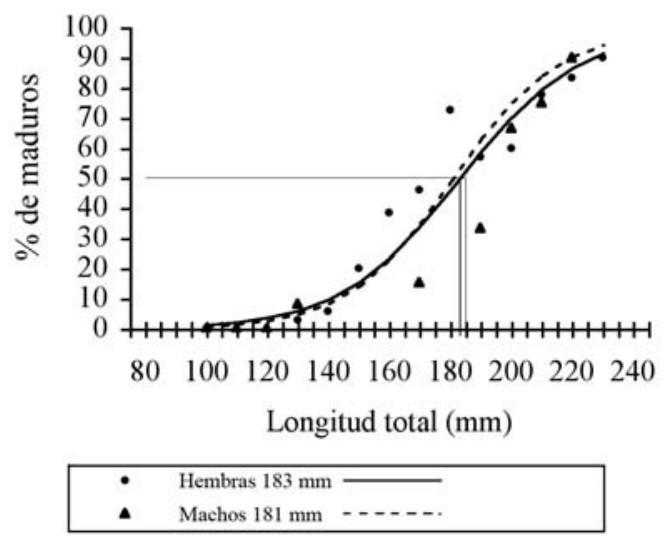

Fig. 9. Porcentaje de organismos maduros de hembras y machos de $P$. grandisquamis colectados en la costa de Jalisco y Colima, México. Los valores fueron ajustados a la ecuación logística.

Fig. 9. Percentage of mature organisms of females and males $P$. grandisquamis collected in the coast of Jalisco and Colima, Mexico. Data were fitted to the logistic equation.

para $P$. grandisquamis oscila entre los $300 \mathrm{y}$ $350 \mathrm{~mm}$ de longitud total; en el presente estudio, la talla máxima encontrada fue de $236 \mathrm{~mm}$, la cual es relativamente inferior a la señalada por los autores antes mencionados, pero superior a la reportada por Amezcua-Linares (1996) para el Pacífico Central de México. 
Mediante la relación talla-peso de los individuos, se ha encontrado que muchas de las especies de peces presentan un tipo de crecimiento isométrico con un valor de la pendiente (b) cercano a tres (Grimes 1987, Ruiz-Ramírez et al. 1997). Sin embargo, también se ha observado que otras especies de peces presentan un tipo de crecimiento alométrico, el cual se caracteriza por tener un valor de $b$ que es estadísticamente diferente de tres. Por la información obtenida en este trabajo, consideramos que $P$. grandisquamis puede presentar un crecimiento de tipo alométrico.

Uno de los factores importantes en la reproducción de los peces es la proporción de hembras y machos que se encuentran en la población; en este estudio la proporción de sexos de $P$. grandisquamis que se encontró fue muy cercana a 1:1 hembra por macho. Sin embargo, lo anterior no siempre ocurre y cuando esta proporción difiere, puede deberse a que en ciertos casos algunos de los sexos tienen mayor supervivencia o a la diferencia que existe en la distribución o al hábitat que prefiere cada uno de estos (Nikolsky 1963, Santamaría-Miranda y Rojas-Herrera 1997, Santamaría et al. 2003a,b, Rojas et al. 2004).

Haciendo un análisis de las tallas promedios encontradas en cada uno de los meses de muestreo y la talla en la que el 50\% de los organismos alcanzan la madurez (183 $\mathrm{mm}$ para hembras y $181 \mathrm{~mm}$ para machos), se observó que durante el periodo de noviembre de 1996 a julio de 1997 se presentaron tallas promedio semejantes a la $\mathrm{L}_{50}$, esto sugiere que la mayoría o por lo menos el $50 \%$ de los individuos se estaban reproduciendo en este periodo. Aunque en los otros años, las tallas promedios mensuales son menores a la $\mathrm{L}_{50}$ encontrada, es muy probable que también se encontraran algunos individuos en actividad reproductiva, aunque en menor proporción; lo anterior se apoya al hacer referencia las tallas que se encontraron en cada uno de los estadios de madurez gonádica.

El IGS se basa en la relación que existe entre el peso de la gónada respecto del peso del organismo y pretende relacionar el grado de desarrollo de la gónada comparativamente con la biomasa total del individuo, ya que las gónadas alcanzan su mayor biomasa antes de que el pez libere las células reproductoras; además este índice ha sido utilizado como un indicador que sirve para señalar el período de reproducción de algunas especies (RodríguezGutiérrez 1992). P. grandisquamis, al parecer se reproduce principalmente en dos periodos del año, uno a mediados y el otro a finales e inicios del año, ya que en estos periodos se encontraron los valores máximos del IGS tanto en las hembras como en los machos.

En la mayoría de los trabajos sobre reproducción, frecuentemente no se considera analizar los valores de IGS en los machos, ya que estos presentan poca variación en comparación con los obtenidos en las hembras. De igual forma, la distribución de los estadios de madurez gonádica en los dos sexos, también sugiere la misma temporada de reproducción señalada (a mitad y final-principio del año), ya que en estos dos periodos se encontraron los porcentajes más altos de hembras y machos con gónadas maduras o en maduración, respectivamente.

El presente informe viene a llenar una laguna en el escaso conocimiento biológico sobre $P$. grandisquamis.

\section{AGRADECIMIENTOS}

Los autores agradecen a la tripulación del barco BIP V, a los profesores y alumnos que participaron en el proyecto: "Demersales" desarrollado en el Centro de Ecología Costera. A Luisa Segura por toda la ayuda proporcionada en el proceso de las gónadas. A Gaspar González por la ayuda en la redacción del resumen en inglés. Dicho proyecto se realizó bajo el permiso de Pesca de Fomento de la SEMARNAP No. 150995-214-03.

\section{RESUMEN}

De junio de 1995 a diciembre de 1998 se realizaron muestreos mensuales y se colectaron en total 492 Pseudupeneus grandisquamis utilizando redes de arrastre sobre fondos blandos en el Pacífico central mexicano (Jalisco y Colima). La relación talla-peso indica que el 
crecimiento es alométrico $\left(p=0.0035 L^{3.46} \mathrm{y} \mathrm{r}^{2}=0.97\right)$. La proporción sexual fue de 1:1.15 (hembras:machos). La talla total promedio fue de $153.8 \mathrm{~mm}$. Se observó una progresión en las tallas de los organismos, esto es, las tallas máximas promedios se capturaron a finales de 1996 y durante 1997. La talla promedio de las hembras $(162 \mathrm{~mm})$ fue mayor a la de los machos (150 mm). En ambos sexos se observaron los primeros cuatro estadios de madurez gonádica; el estadio mas frecuente en las hembras fue el IV (gónadas maduras, $48.6 \%$ ) y en los machos fue el III (gónadas en maduración, $45.0 \%$ ). Se identificaron siete etapas microscópicas de desarrollo de los ovocitos, para los cuales se registró un desarrollo de tipo asincrónico. Los testículos maduros presentaron espermatozoides dentro del lumen lobular y en el conducto. Los valores máximos del índice gonadosomático, así como los mayores porcentajes de individuos con gónadas maduras (a nivel macroscópico y microscópico), fueron observados en invierno y verano, sugiriendo que esta especie tiene dos picos de reproducción por año. La longitud en la que el 50\% de los individuos presento gónadas en maduración $\left(L_{50}\right)$ fue de $183 \mathrm{~mm}$ para las hembras y $181 \mathrm{~mm}$ para los machos.

Palabras clave: Pseudupeneus grandisquamis, Mullidae, reproducción de peces, Jalisco, Colima, México.

\section{REFERENCIAS}

Aguilar-Palomino, B., J. Mariscal-Romero, G. GonzálezSansón \& L.E. Rodríguez-Ibarra. 1996. Ictiofauna demersal de fondos blandos de la plataforma continental de Jalisco y Colima, México, en la primavera de 1995. Cien. Mar. 22: 469-481.

Allen, G. R. \& D.R. Robertson. 1994. Fishes of the Tropical Eastern Pacific. University of Hawaii. Honolulu, Hawaii, EEUU. 174 p.

Amezcua-Linares, F. 1996. Peces demersales de la plataforma continental del Pacífico Central de México. Instituto de Ciencias de Mar y Limnología, Universidad Nacional Autónoma de México y Comisión Nacional para el Conocimiento y Uso de la Biodiversidad, México, D.F. 184 p.

Cole, K.S. 2002. Gonad morphology, sexual development, and colony composition in the obligate coral-dwelling damselfish Dascyllus aruanus. Mar. Biol. 140: 151-163.

De la Lanza-Espino, G. 1991.Oceanografía de mares mexicanos. AGT, México, D.F. 569 p.

Echeverria, T.W. 1987. Thirty-four species of California rockfishes: maturity and seasonality of reproduction. Fish. Bull. 85: 229-250.
Filonov A.E., Y.E. Tereshchenko, C.O. Monzón, M.E. González-Ruelas \& E. Godínez-Domínguez. 2000. Variabilidad estacional de campos de temperatura y salinidad en la zona costera de los estados de Jalisco y Colima. Cien. Mar. 25: 303-321.

Gill, T.N. 1863. Descriptive enumeration of a collection of fishes from the western coast of Central America, presented to the Smithsonian Institution by Captain John M. Dow. Proc. Acad. Nat. Sci. Phila. 162-174.

Godínez-Domínguez, E. \& G. González-Sansón. 1998. Variación de los patrones de distribución batimétrica de la fauna macrobentónica en la plataforma continental de Jalisco y Colima, México. Cien. Mar. 24: 337-351.

González-Sansón, G., B. Aguilar-Palomino, J. ArciniegaFlores, R. García de Quevedo-Machain, E. GodínezDomínguez, V. Landa-Jaime, J. Mariscal-Romero, J.E. Michel-Morfín \& M. Saucedo-Lozano. 1997. Variación estacional de la abundancia de la fauna de fondos blandos en la plataforma continental de Jalisco y Colima, México. (Primavera 1995). Cien. Mar. 23: 93-110.

Grimes, C.B. 1987. Reproductive biology of the Lutjanidae: A review. p. 239-294. In J.J. Polovina \& S. Ralston Tropical (eds.). Snappers and grupers: Biology and Fisheries management. Westview, Boulder, Colorado, EEUU.

Landa-Jaime, V., J. Arciniega Flores, R. García de Quevedo, J.E. Michel-Morfín \& G. González-Sansón. 1997. Crustáceos decápodos y estomatópodos de fondos blandos de la plataforma continental de Jalisco y Colima, México. Cien. Mar. 23: 403-417.

Lucano-Ramírez, G. 1998. Ciclo reproductivo de Lutjanus peru (Nichols y Murphy, 1922) (Pisces:Lutjanidae) en las costa sur de Jalisco, México. Tesis de maestría. Universidad Nacional Autónoma de México, México, D.F. 83 p.

Lucano-Ramírez, G., M. Villagran-Santa Cruz, S. RuizRamírez \& T. López-Murillo. 2001. Histología de los ovocitos de Lutjanus peru (Nichols y Murphy, 1922) (Picses: Lutjanidae). Cien. Mar. 27: 335-349.

Nikolsky, G. 1963. The ecology of fishes. Academic, Londres. 352 p.

Rodríguez-Gutiérrez, M. 1992. Técnicas de evaluación cuantitativa de la madurez gonádica en peces. AGT, México, D.F. 79 p.

Rojas, M., J.R, E. Maravilla \& F. Chicas B. 2004. Hábitos alimentarios del pargo mancha Lutjanus guttatus (Pisces: Lutjanidae) en Los Cóbanos y Puerto La Libertad, El Salvador. Rev. Biol. Trop. 52: 163-170. 
Ruiz-Ramírez, S., G. Lucano-Ramírez \& J. MariscalRomero. 1997. Length-Weight relationships of softbottom demersal fishes from Jalisco and Colima states, Mexico. Naga, ICLARM Q. 20: 62-63.

Schneider, M. 1995. Mullidae. Salmonetes. In W. Fischer, F. Krupp, W. Schneider, C. Sommer, K.E. Carpenter, \& V. Niem (eds.), Guía FAO para la identificación de especies para los fines de la pesca. Pacífico Centro-Oriental. Volumen III. Vertebrados Parte 2. FAO, Roma.

Santamaría-Miranda, A. \& A.A. Rojas-Herrera. 1997. Análisis de la variación morfológica del huachinango, Lutjanus peru (Nichols y Murphy, 1922) (Pisces: Lutjanidae) en el litoral de la costa chica de Guerrero, México. Periodo noviembre 1992-diciembre 1994. Sociedad Ictiológica Mexicana. Resúmenes del V Congreso Nacional de Ictiología. Mazatlán, Sinaloa, México. 170 p.
Santamaría-Miranda, A., J.F. Elorduy-Garay, M. VillalejoFuerte \& A.A. Rojas-Herrera. 2003a. Desarrollo gonadal y ciclo reproductivo de Lutjanus peru (Pisces: Lutjanidae) en Guerrero, México. Rev. Biol. Trop. 51: 489-502.

Santamaría-Miranda, A., J.F. Elorduy-Garay \& A.A. Rojas Herrera. 2003b. Hábitos alimentarios de Lutjanus peru (Pisces: Lutjanidae) en las costas de Guerrero, México. Rev. Biol. Trop. 51: 503-518.

Zar, J.H. 1996. Bioestatistical Analysis. Prentice Hall, Nueva Jersey, EEUU. X + 662 p.

\section{REFERENCIA DE INTERNET}

Froese, R. \& D. Pauly (eds). 2005. FishBase. World Wide Web electronic publication. www.fishbase.org (consultado 1-V-2005). 
